Modeling, Identification and Control, Vol. 28, No. 1, 2007, pp. 19-30

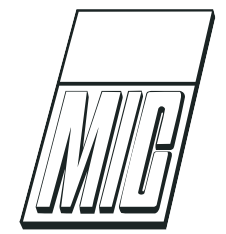

\title{
Kinematic Models for Manoeuvring and Seakeeping of Marine Vessels
}

\author{
Tristan Perez, ${ }^{1}$ Thor I. Fossen ${ }^{2}$ \\ ${ }^{1}$ Centre for Ships and Ocean Structures, Norwegian University of Science and Technology, NO-7941 Trondheim, \\ Norway. E-mail: tristan.perez@ntnu.no \\ ${ }^{2}$ Department of Engineering Cybernetics, Norwegian University of Science and Technology, NO-7491 Trondheim, \\ Norway.
}

\begin{abstract}
The motion of marine vessels has traditionally been studied using two different approaches: manoeuvring and seakeeping. These two approaches use different reference frames and coordinate systems to describe the motion. This paper derives the kinematic models that characterize the transformation of motion variables (position, velocity, accelerations) and forces between the different coordinate systems used in these theories. The derivations hereby presented are done in terms of the formalism adopted in robotics. The advantage of this formulation is the use of matrix notation and operations. As an application, the transformation of linear equations of motion used in seakeeping into body-fixed coordinates is considered for both zero and forward speed.
\end{abstract}

Keywords: Kinematics, ship motion, seakeeping, manoeuvring.

\section{Introduction}

The study of ship dynamics has traditionally been covered by two main theories: Manoeuvring and Seakeeping. Manoeuvring refers to the study of ship motion in the absence of wave excitation (calm water). Seakeeping, on the other hand, refers to the study of motion when there is wave excitation and while the vessel keeps its course and its speed constant (which includes the case of zero speed). Although both areas are concerned with the same issues: study of motion, stability and control, the separation allows making different assumptions that simplify the study in each case. A chief distinguishing characteristic of these theories is the use of different coordinates and reference systems to express the equations of motion.

In manoeuvring, the equations of motion are described relative to a coordinate system fixed to the vessel (a body-fixed coordinate system), whereas in seakeeping the motion is described relative to a coordinate system fixed to an equilibrium virtual vessel that moves at a constant speed and heading corresponding the average motion of the actual vessel. Most hydrodynamic programs compute radiation and wave excitation forces in this equilibrium frame.

When time-domain simulations and motion control system designs are considered, it is necessary to use a unified framework, and a body-fixed coordinate system is a natural choice. This calls for kinematic models that characterize the transformation of motion variables (position, velocity and accelerations) and forces between different coordinate systems. For the case of a vessel operating at zero forward speed, the equilibrium coordinate system can be considered fixed to the earth. The kinematic models then describe the traditional transformation between body- and earth-fixed coordinates used in robotics and aeronautics. For the case of forward speed, transformations between earthfixed, body-fixed and equilibrium (or seakeeping) coordinates are different. Details of the latter transfor- 
mations are often only outlined or considered for small angles in the literature - see, for example, Bishop and Price (1981); Lloyd (1989); Graham (1990); Faltinsen (2005).

Therefore, this paper reviews the kinematic models commonly used in both theories and provides a complete derivation of the transformations that link these descriptions. In recent years, there has been a significant interest of these models in relation to the timedomain models for simulation and control system design based on data obtained from seakeeping programs, and unified models for manoeuvring and seakeepingsee Bailey et al. (1997); Fossen and Smogeli (2004); Fossen (2005); Smogeli et al. (2005); Perez (2005); Perez and Fossen (2006).

\section{Coordinate Systems}

To describe the position and orientation of a ship, the following dextral orthogonal coordinate systems are commonly used - see Figure 1:

- North-East-Down, $\{n\}$;

- $\operatorname{Body}(-$ fixed), $\{b\}$;

- Seakeeping, $\{s\}$.

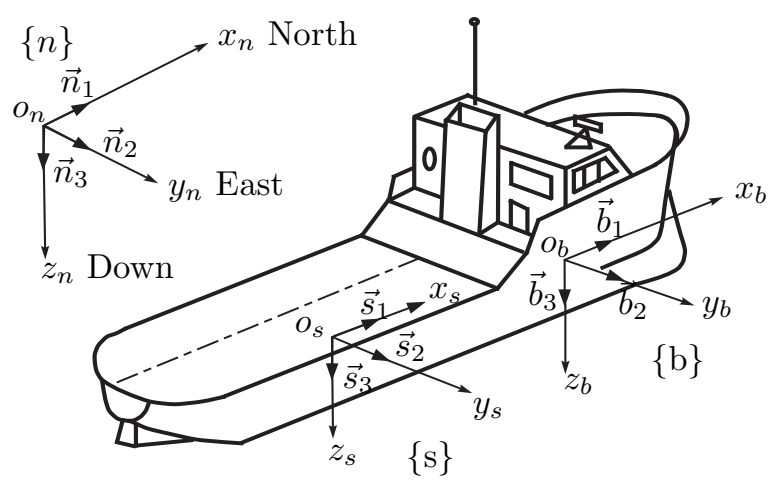

Figure 1: Coordinate systems used for ship motion description.

The North-East-Down coordinate system $\{n\} \equiv\left(o_{n}, \vec{n}_{1}, \vec{n}_{2}, \vec{n}_{3}\right)$ is the local geographic coordinate system fixed to the Earth. This is usually determined by a tangent plane attached to the geodetic reference ellipsoid (WGS84) at a point of interest, which determines the origin of the system $o_{n}$. The positive unit vector $\vec{n}_{1}$ points towards the true North, $\vec{n}_{2}$ points towards the East, and $\vec{n}_{3}$ points towards the interior of the earth perpendicular to the reference ellipsoid completing the dextral orthogonal system.
The Body-fixed coordinate system $\{b\} \equiv$ $\left(o_{b}, \vec{b}_{1}, \vec{b}_{2}, \vec{b}_{3}\right)$ is fixed to the vessel. The positive unit vector $\vec{b}_{1}$ points towards the bow, $\vec{b}_{2}$ points towards starboard and $\vec{b}_{3}$ points downwards completing the dextral orthogonal system. For marine vehicles, the origin $o_{b}$ is usually chosen to be amidships $(L p p / 2)$ and at the intersection of the longitudinal plane of symmetry $(x-z)$ and the design water line. Different applications, however, may require other locations for $o_{b}$.

The Seakeeping coordinate systems $\{s\} \equiv$ $\left(o_{s}, \vec{s}_{1}, \vec{s}_{2}, \vec{s}_{3}\right)$ moves at the average speed of the vessel following its path. This coordinate system can only be considered when the vessel sails with a straight average path at a constant average speed. That is, this coordinate system is not fixed to the vessel but to an equilibrium state or virtual vessel, and the action of waves makes the vessel oscillate about this equilibrium; this is illustrated in Figure 2. The positive unit vector $\vec{s}_{1}$ points forward and is aligned with the average velocity vector. The positive unit vector $\vec{s}_{2}$ points towards starboard, and $\vec{s}_{3}$ points downwards completing the dextral orthogonal system. The origin $o_{s}$ is usually determined such that the $z_{s}$-axis passes through the equilibrium position of the centre of gravity of the vessel, and the horizontal plane that contains $\vec{s}_{1}$ and $\vec{s}_{2}$ coincides with the mean free surface of the water.

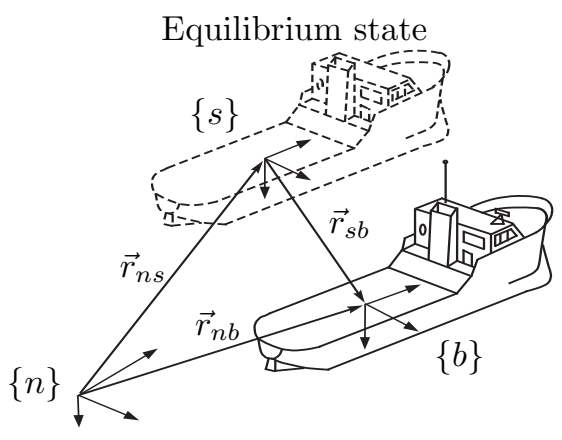

Figure 2: Coordinate systems and relative positions.

The coordinate system $\{n\}$ is used to define the position of the vessel on the earth, and the direction of wind, waves and current. This frame is considered inertial, which is a reasonable assumption because the velocity of marine vehicles is relatively small, and thus the forces due to the rotation of the Earth are negligible relative to the hydrodynamic forces acting on the vehicle. The coordinate system $\{b\}$ is used to express velocity and acceleration measurements taken on board. The equations of motion of the vessel are normally formulated about the point $o_{b}$. This coordinate system is also used to define some ship motion performance indices - see Lloyd (1989). The coordinate 
system $\{s\}$ is used to define the wave elevation at the vessel's average location and to compute the hydrodynamic forces using standard software based on hydrodynamic theories - see, for example, Fathi (2004), Journee and Adegeest (2003), WAMIT (2004). The positive convention described above for the coordinate systems $\{n\},\{b\}$ and $\{s\}$ will be the one adopted in the rest of the paper. In the literature and in different hydrodynamic programs, however, other conventions may be used.

\section{Mathematical Background}

This section defines the mathematical notation and summarizes some key results that will be used in the subsequent sections. The material has been adapted from Sciavicco and Siciliano (2004), Egeland and Gravdahl (2002), Kane and Levinson (1985) and Rao (2006).

\subsection{Coordinate Systems and Vector Notation}

We will use the notation $\vec{u}$ to refer to a coordinate free vector, i.e., a directed line segment. When a vector is described relative to a coordinate system $\{a\}$, we will use the following representation:

$$
\vec{u}=u_{1}^{a} \vec{a}_{1}+u_{2}^{a} \vec{a}_{2}+u_{3}^{a} \vec{a}_{3},
$$

where $\vec{a}_{i}$ are the unit vectors that define $\{a\}, u_{i}^{a}$ are the measures of $\vec{u}$ along $\vec{a}_{i}$, and $u_{i}^{a} \vec{a}_{i}$ are the components of $\vec{u}$ in $\{a\}$. We will also use the coordinate form of $\vec{u}$ in $\{a\}$ which is represented as a column vector in $\mathbb{R}^{3}$ :

$$
\mathbf{u}^{a} \triangleq\left[u_{1}^{a}, u_{2}^{a}, u_{3}^{a}\right]^{T} .
$$

This representation enables the use of matrix notation and operations to discuss different properties of vector quantities. The coordinate form of a vector is a representation relative to a particular basis, whereas the vector itself is basis independent-coordinate free.

When discussing properties that hold regardless of the chosen basis, we will simply use u. For example, $\vec{u} \cdot \vec{v}=\mathbf{u}^{T} \mathbf{v}$, which holds in any basis as long as both coordinate vectors are given with respect to the same basis: $\vec{u} \cdot \vec{v}=\left(\mathbf{u}^{a}\right)^{T} \mathbf{v}^{a}=\left(\mathbf{u}^{b}\right)^{T} \mathbf{v}^{b}$.

The cross-product of two vectors $\vec{c}=\vec{a} \times \vec{b}$ can be represented in coordinate form as

$$
\mathbf{c}=\mathbf{S}(\mathbf{a}) \mathbf{b},
$$

where the matrix $\mathbf{S}(\mathbf{a})$ gives the skew-symmetric form of a coordinate vector $\mathbf{a}=\left[a_{1}, a_{2}, a_{3}\right]^{T}$ :

$$
\mathbf{S}(\mathbf{a}) \triangleq\left[\begin{array}{ccc}
0 & -a_{3} & a_{2} \\
a_{3} & 0 & -a_{1} \\
-a_{2} & a_{1} & 0
\end{array}\right]
$$

Note that $\mathbf{S}(\mathbf{a})=-\mathbf{S}^{T}(\mathbf{a})$.

\subsection{Rotation Matrix}

The transformation of coordinate vectors between different frames is performed via appropriate transformation matrices. The generic unbound vector $\vec{r}$, can be expressed in either $\{a\}$ or $\{b\}$ as

$$
\vec{r}=\sum_{i=1}^{3} r_{i}^{a} \vec{a}_{i}=\sum_{i=1}^{3} r_{i}^{b} \vec{b}_{i} .
$$

Then, the rotation matrix that takes $\{a\}$ into the orientation of $\{b\}$ is defined as

$$
\mathbf{R}_{b}^{a} \triangleq\left[R_{i j}=\vec{a}_{i} \cdot \vec{b}_{j}\right]
$$

Note that $\mathbf{R}_{b}^{a}$ transforms coordinate vectors in $\{b\}$ to coordinate vectors in $\{a\}$ :

$$
\mathbf{r}^{a}=\mathbf{R}_{b}^{a} \mathbf{r}^{b}
$$

Rotation matrices are elements of the special orthogonal group of order 3, $S O(3): \mathbf{R R}^{T}=\mathbf{I}_{3 \times 3}$, and $\operatorname{det}(\mathbf{R})=1$. Thus, $\mathbf{R}^{-1}=\mathbf{R}^{T}$.

A rotation is called simple, if it is a rotation about a single axis (not necessarily a coordinate axis). If we consider simple rotations about the coordinates axes, however, we obtain:

- A rotation of an angle $\psi$ about the $z$-axis:

$$
\mathbf{R}_{z, \psi}=\left[\begin{array}{ccc}
\cos \psi & -\sin \psi & 0 \\
\sin \psi & \cos \psi & 0 \\
0 & 0 & 1
\end{array}\right]
$$

- A rotation of an angle $\theta$ about the $y$-axis:

$$
\mathbf{R}_{y, \theta}=\left[\begin{array}{ccc}
\cos \theta & 0 & \sin \theta \\
0 & 1 & 0 \\
-\sin \theta & 0 & \cos \theta
\end{array}\right]
$$

- A rotation of an angle $\phi$ about the $x$-axis:

$$
\mathbf{R}_{x, \phi}=\left[\begin{array}{ccc}
1 & 0 & 0 \\
0 & \cos \phi & -\sin \phi \\
0 & \sin \phi & \cos \phi
\end{array}\right]
$$

The above results follow from (6).

\subsection{Euler Angles: Roll, Pitch and Yaw}

The attitude (or orientation) of a coordinate system $\{b\}$ relative to $\{a\}$ can be expressed by three consecutive rotations about the main axes that take $\{a\}$ into $\{b\}$. These rotations can be performed in a different 
order (there are 12 different ways of doing this), and each triplet of rotated angles is called a set of Euler angles. The set most commonly used in navigation is that of roll, pitch and yaw, which corresponds to the rotations performed in the following order:

1. Rotation about the $z$-axis of $\{a\}$ an angle $\psi$ (yaw angle) resulting in the frame $\left\{a^{\prime}\right\}$;

2. Rotation about the $y$-axis of $\left\{a^{\prime}\right\}$ an angle $\theta$ (pitch angle) resulting in the frame $\left\{a^{\prime \prime}\right\}$;

3. Rotation about the $x$-axis of $\left\{a^{\prime \prime}\right\}$ an angle $\phi$ (roll angle) resulting in $\{b\}$.

The positive angle convention corresponds to a righthanded screw advancing in the positive direction of the axis of rotation.

Using these consecutive single rotations, a rotation matrix can be expressed as

$$
\mathbf{R}_{b}^{a}=\mathbf{R}_{z, \psi} \mathbf{R}_{y^{\prime}, \theta} \mathbf{R}_{x^{\prime \prime}, \phi} .
$$

After multiplication

$$
\begin{aligned}
& \mathbf{R}_{b}^{a}= \\
& {\left[\begin{array}{ccc}
c_{\psi} c_{\theta} & -s_{\psi} c_{\phi}+c_{\psi} s_{\theta} s_{\phi} & s_{\psi} s_{\phi}+c_{\psi} c_{\phi} s_{\theta} \\
s_{\psi} c_{\theta} & c_{\psi} c_{\phi}+s_{\phi} s_{\theta} s_{\psi} & -c_{\psi} s_{\phi}+s_{\psi} c_{\phi} s_{\theta} \\
-s_{\theta} & c_{\theta} s_{\phi} & c_{\theta} c_{\phi}
\end{array}\right],}
\end{aligned}
$$

where $s_{x} \equiv \sin (x)$ and $c_{x} \equiv \cos (x)$. Note that the matrix multiplication order in (11) is consistent with (7).

\subsection{Angular Velocity}

Since the rotation matrix $\mathbf{R}_{b}^{a}$ is orthogonal $\left(\mathbf{R}_{b}^{a}\left(\mathbf{R}_{b}^{a}\right)^{T}=I\right)$, then

$$
\frac{d}{d t}\left[\mathbf{R}_{b}^{a}\left(\mathbf{R}_{b}^{a}\right)^{T}\right]=\dot{\mathbf{R}}_{b}^{a}\left(\mathbf{R}_{b}^{a}\right)^{T}+\mathbf{R}_{b}^{a}\left(\dot{\mathbf{R}}_{b}^{a}\right)^{T}=\mathbf{0}
$$

This implies that $\dot{\mathbf{R}}_{b}^{a}\left(\mathbf{R}_{b}^{a}\right)^{T}$ is a skew-symmetric matrix, and thus it can be described by a column vector as in (4). The vector $\boldsymbol{\omega}_{a b}^{a}$ of angular velocity of the frame $\{b\}$ with respect to the frame $\{a\}$, with coordinates in the frame $\{a\}$ is defined such that

$$
\boldsymbol{\omega}_{a b}^{a}: \quad \mathbf{S}\left(\boldsymbol{\omega}_{a b}^{a}\right)=\dot{\mathbf{R}}_{b}^{a}\left(\mathbf{R}_{b}^{a}\right)^{T}
$$

It also follows that

$$
\dot{\mathbf{R}}_{b}^{a}=\mathbf{S}\left(\boldsymbol{\omega}_{a b}^{a}\right) \mathbf{R}_{b}^{a}=\mathbf{R}_{b}^{a} \mathbf{S}\left(\boldsymbol{\omega}_{a b}^{b}\right) .
$$

If we further consider three composite rotations $\mathbf{R}_{d}^{a}=$ $\mathbf{R}_{b}^{a} \mathbf{R}_{c}^{b} \mathbf{R}_{d}^{c}$, then

$$
\dot{\mathbf{R}}_{d}^{a}=\dot{\mathbf{R}}_{b}^{a} \mathbf{R}_{c}^{b} \mathbf{R}_{d}^{c}+\mathbf{R}_{b}^{a} \dot{\mathbf{R}}_{c}^{b} \mathbf{R}_{d}^{c}+\mathbf{R}_{b}^{a} \mathbf{R}_{c}^{b} \dot{\mathbf{R}}_{d}^{c} .
$$

Using (14), we find that

$$
\begin{aligned}
& \mathbf{S}\left(\boldsymbol{\omega}_{a d}^{a}\right)=\dot{\mathbf{R}}_{d}^{a}\left(\mathbf{R}_{d}^{a}\right)^{T} \\
& =\left(\dot{\mathbf{R}}_{b}^{a} \mathbf{R}_{c}^{b} \mathbf{R}_{d}^{c}+\mathbf{R}_{b}^{a} \dot{\mathbf{R}}_{c}^{b} \mathbf{R}_{d}^{c}+\mathbf{R}_{b}^{a} \mathbf{R}_{c}^{b} \dot{\mathbf{R}}_{d}^{c}\right)\left(\mathbf{R}_{d}^{c}\right)^{T}\left(\mathbf{R}_{c}^{b}\right)^{T}\left(\mathbf{R}_{b}^{a}\right)^{T} \\
& =\dot{\mathbf{R}}_{b}^{a}\left(\mathbf{R}_{b}^{a}\right)^{T}+\mathbf{R}_{b}^{a} \dot{\mathbf{R}}_{c}^{b}\left(\mathbf{R}_{c}^{b}\right)^{T}\left(\mathbf{R}_{b}^{a}\right)^{T}+\mathbf{R}_{c}^{a} \dot{\mathbf{R}}_{d}^{c}\left(\mathbf{R}_{d}^{c}\right)^{T}\left(\mathbf{R}_{c}^{a}\right)^{T} \\
& =\mathbf{S}\left(\boldsymbol{\omega}_{a b}^{a}\right)+\mathbf{R}_{b}^{a} \mathbf{S}\left(\boldsymbol{\omega}_{b c}^{b}\right)\left(\mathbf{R}_{b}^{a}\right)^{T}+\mathbf{R}_{c}^{a} \mathbf{S}\left(\boldsymbol{\omega}_{c d}^{c}\right)\left(\mathbf{R}_{c}^{a}\right)^{T} \\
& =\mathbf{S}\left(\boldsymbol{\omega}_{a b}^{a}\right)+\mathbf{S}\left(\boldsymbol{\omega}_{b c}^{a}\right)+\mathbf{S}\left(\boldsymbol{\omega}_{c d}^{a}\right),
\end{aligned}
$$

which from the linearity of $\mathbf{S}(\cdot)$ implies that

$$
\boldsymbol{\omega}_{a d}^{a}=\boldsymbol{\omega}_{a b}^{a}+\boldsymbol{\omega}_{b c}^{a}+\boldsymbol{\omega}_{c d}^{a} .
$$

This holds in any coordinate system and for any number of consecutive rotations (Kane and Levinson, 1985):

$$
\vec{\omega}_{a d}=\vec{\omega}_{a b}+\vec{\omega}_{b c}+\vec{\omega}_{c d}
$$

\subsection{Relationship Between Angular Velocity and Euler-angle Derivatives}

Let us consider the rotation from $\{a\}$ to $\{d\}$ via simple roll, pitch and yaw rotations:

$$
\mathbf{R}_{b}^{a}=\mathbf{R}_{z, \psi}, \quad \mathbf{R}_{c}^{b}=\mathbf{R}_{y, \theta}, \quad \mathbf{R}_{d}^{c}=\mathbf{R}_{x, \phi} .
$$

The angular velocities corresponding to these rotations are

$$
\begin{aligned}
\boldsymbol{\omega}_{a b}^{a} & =[0,0, \dot{\psi}]^{T}, \\
\boldsymbol{\omega}_{b c}^{b} & =[0, \dot{\theta}, 0]^{T}, \\
\boldsymbol{\omega}_{c d}^{c} & =[\dot{\phi}, 0,0]^{T} .
\end{aligned}
$$

Using (19), we can write

$$
\boldsymbol{\omega}_{a d}^{a}=\boldsymbol{\omega}_{a b}^{a}+\mathbf{R}_{b}^{a} \boldsymbol{\omega}_{b c}^{b}+\mathbf{R}_{b}^{a} \mathbf{R}_{c}^{b} \boldsymbol{\omega}_{c d}^{c},
$$

and

$$
\boldsymbol{\omega}_{a d}^{d}=\mathbf{R}_{a}^{d} \boldsymbol{\omega}_{a b}^{a}+\mathbf{R}_{c}^{d} \mathbf{R}_{b}^{c} \boldsymbol{\omega}_{b c}^{b}+\mathbf{R}_{c}^{c} \boldsymbol{\omega}_{c d}^{c} .
$$

Define the vector of roll, pitch and yaw associated with the rotation from $\{a\}$ to $\{d\}$ as

$$
\boldsymbol{\Theta}_{a d} \triangleq[\phi, \theta, \psi]^{T} \text {. }
$$

Then (22) and (23) can be expressed as

$$
\begin{gathered}
\boldsymbol{\omega}_{a d}^{a}=\mathbf{T}_{a}^{-1}\left(\boldsymbol{\Theta}_{a d}\right) \dot{\boldsymbol{\Theta}}_{a d}=\left[\begin{array}{ccc}
c_{\psi} c_{\theta} & -s_{\psi} & 0 \\
s_{\psi} c_{\theta} & c_{\psi} & 0 \\
-s_{\theta} & 0 & 1
\end{array}\right] \dot{\boldsymbol{\Theta}}_{a d} \\
\boldsymbol{\omega}_{a d}^{d}=\mathbf{T}_{d}^{-1}\left(\boldsymbol{\Theta}_{a d}\right) \dot{\boldsymbol{\Theta}}_{a d}=\left[\begin{array}{ccc}
1 & 0 & -s_{\theta} \\
0 & c_{\phi} & s_{\phi} c_{\theta} \\
0 & -s_{\phi} & c_{\phi} c_{\theta}
\end{array}\right] \dot{\boldsymbol{\Theta}}_{a d}
\end{gathered}
$$

The inverse relationships are

$$
\dot{\boldsymbol{\Theta}}_{a d}=\mathbf{T}_{a}\left(\boldsymbol{\Theta}_{a d}\right) \boldsymbol{\omega}_{a d}^{a}=\left[\begin{array}{ccc}
\frac{c_{\psi}}{c_{\theta}} & \frac{s_{\psi}}{c_{\theta}} & 0 \\
-s_{\psi} & c_{\psi} & 0 \\
c_{\psi} t_{\theta} & s_{\psi} t_{\theta} & 1
\end{array}\right] \boldsymbol{\omega}_{a d}^{a}
$$




$$
\dot{\boldsymbol{\Theta}}_{a d}=\mathbf{T}_{d}\left(\boldsymbol{\Theta}_{a d}\right) \boldsymbol{\omega}_{a d}^{d}=\left[\begin{array}{ccc}
1 & s_{\phi} t_{\theta} & c_{\phi} t_{\theta} \\
0 & c_{\phi} & -s_{\phi} \\
0 & \frac{s_{\phi}}{c_{\theta}} & \frac{c_{\phi}}{c_{\theta}}
\end{array}\right] \boldsymbol{\omega}_{a d}^{d}
$$

Note that $\operatorname{det}\left(\mathbf{T}_{a}\right)=\operatorname{det}\left(\mathbf{T}_{d}\right)=1 / \cos \theta$, and that $\mathbf{T}_{a}^{-1} \neq \mathbf{T}_{a}^{T}$ and $\mathbf{T}_{d}^{-1} \neq \mathbf{T}_{d}^{T}$. Therefore, neither $\mathbf{T}_{a}$ nor $\mathbf{T}_{d}$ are orthogonal. Also, $\mathbf{T}_{a}^{-1}$ and $\mathbf{T}_{d}^{-1}$ are singular for $\theta= \pm \pi / 2$ - known as the Euler-angle singularity.

\subsection{Position and Velocity Coordinate Vectors}

For position and velocity coordinate vectors we will use a three-script notation. For example, if $\vec{r}_{a b}$ is the vector from $\{a\}$ to $\{b\}$, which indicates the position of $\{b\}$ relative to $\{a\}$. Then, in coordinate form, the notation $\mathbf{r}_{a b}^{a}$ indicates the position of $\{b\}$ relative to $\{a\}$ expressed in $\{a\}$. That is, the upper script indicates in which coordinate system the vector is expressed, while the lower scripts indicate the point of interest and the coordinate system with respect to which the position is considered. This vector is expressed in $\{b\}$, for example, can be obtained from

$$
\mathbf{r}_{a b}^{b}=\mathbf{R}_{a}^{b} \mathbf{r}_{a b}^{a}
$$

Then, the notation $\mathbf{v}_{a b}^{a}$ indicates the velocity of $\{b\}$ relative to $\{a\}$ expressed in $\{a\}$. We will use this notation only when the frame with respect to which the derivative is taken is inertial, i.e., if $a$ is inertial then,

$$
\mathbf{v}_{a b}^{a} \triangleq \dot{\mathbf{r}}_{a b}^{a} \equiv \frac{{ }^{a} d}{d t} \vec{r}_{a b} .
$$

The derivative of a vector must be specified with respect to a particular coordinate system. We should then be careful when taking derivatives in coordinate form because the information about the reference frames in which the derivative is taken can be lost. For example, the following is a well known relationship between the derivatives of a vector in two coordinate systems (Kane and Levinson, 1985):

$$
\frac{{ }^{a} d \vec{r}}{d t}=\frac{{ }^{b} d \vec{r}}{d t}+\vec{\omega}_{a b} \times \vec{r},
$$

in which

$$
\begin{aligned}
& \frac{{ }^{a} d \vec{r}}{d t}=\dot{r}_{1}^{a} \vec{a}_{1}+\dot{r}_{2}^{a} \vec{a}_{2}+\dot{r}_{3}^{a} \vec{a}_{3}, \\
& \frac{{ }^{b} d \vec{r}}{d t}=\dot{r}_{1}^{b} \vec{b}_{1}+\dot{r}_{2}^{b} \vec{b}_{2}+\dot{r}_{3}^{b} \vec{b}_{3} .
\end{aligned}
$$

This relationship can be derived in coordinate form by considering

$$
\mathbf{r}^{a}=\mathbf{R}_{b}^{a} \mathbf{r}^{b}
$$

Taking the time derivative of (31), gives

$$
\dot{\mathbf{r}}^{a}=\mathbf{R}_{b}^{a}\left[\dot{\mathbf{r}}^{b}+\mathbf{S}\left(\boldsymbol{\omega}_{a b}^{b}\right) \mathbf{r}^{b}\right]
$$

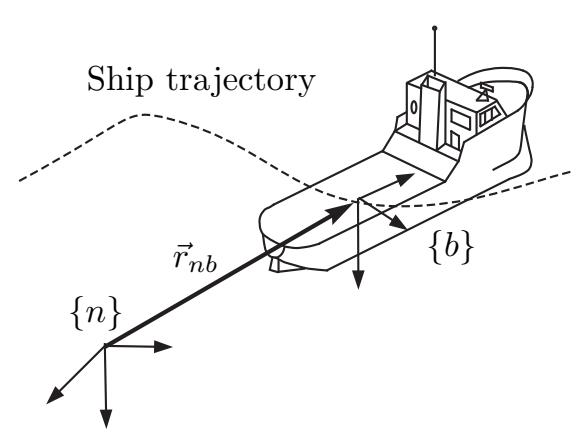

Figure 3: Ship position used in manoeuvring.

However, it should be noted here that the derivative on the left-hand side is taken with respect to the coordinate system $\{a\}$ while the derivative on the right-hand side is taken with respect to the coordinate system $\{b\}$. To express the above in $\{b\}$, we would have to multiply both sides by $\mathbf{R}_{a}^{b}$, which would lead to

$$
\dot{\mathbf{r}}^{b}=\dot{\mathbf{r}}^{b}+\mathbf{S}\left(\boldsymbol{\omega}_{a b}^{b}\right) \mathbf{r}^{b}
$$

This should be interpreted as

$$
{ }^{a} \dot{\mathbf{r}}^{b}={ }^{b} \dot{\mathbf{r}}^{b}+\mathbf{S}\left(\boldsymbol{\omega}_{a b}^{b}\right) \mathbf{r}^{b}
$$

where the upper-left script indicates the coordinate system with respect to which the derivative is taken. The above expression is the coordinate form of (29).

\section{Manoeuvring Theory and Kinematic Models}

\subsection{Coordinate Systems and Motion Variables}

In manoeuvring theory, the position of a ship is given by the position of the origin of $\{b\}$ relative to $\{n\}$ : $\vec{r}_{n b}$-Figure 3 illustrates this. When expressed in coordinate form in $\{n\}$, this vector gives the North, East and Down positions:

$$
\mathbf{r}_{n b}^{n} \triangleq[N, E, D]^{T}
$$

The attitude of the vessel will be given by the angles or roll, pitch and yaw that take $\{n\}$ into $\{b\}$ :

$$
\boldsymbol{\Theta}_{n b} \triangleq[\phi, \theta, \psi]^{T}
$$

The linear and angular velocities are more conveniently expressed in the body-fixed coordinate system. The vessel linear velocity in $\{b\}$ is given by

$$
\mathbf{v}_{n b}^{b} \triangleq \mathbf{R}_{n}^{b} \dot{\mathbf{r}}_{n b}^{n},=\mathbf{R}_{n}^{b}[\dot{N}, \dot{E}, \dot{D}]^{T},
$$


The components of $\mathbf{v}_{n b}^{b}$ will be denoted by

$$
\mathbf{v}_{n b}^{b}=[u, v, w]^{T},
$$

which are called surge, sway and heave speeds respectively (SNAME, 1950). Note that

$$
\int_{0}^{t} \mathbf{v}_{n b}^{b} d \tau
$$

has no physical meaning; the vessel trajectory is given by

$$
\mathbf{r}_{n b}^{n}(t)=\int_{0}^{t} \mathbf{R}_{b}^{n} \mathbf{v}_{n b}^{b} d \tau+\mathbf{r}_{n b}^{n}(0)
$$

The angular velocity in $\{b\}$ is given by

$$
\boldsymbol{\omega}_{n b}^{b}=[p, q, r]^{T}: \quad \dot{\mathbf{R}}_{b}^{n}=\mathbf{R}_{b}^{n} \mathbf{S}\left(\boldsymbol{\omega}_{n b}^{b}\right),
$$

which follows from (15). The components are called roll, pitch and yaw rate respectively (SNAME, 1950). Note that the relationship between $\boldsymbol{\omega}_{n b}^{b}$ and the time derivative of the Euler angles follows from (28) modulo substitution $a, d$ by $n, b$ :

$$
\dot{\boldsymbol{\Theta}}_{n b}=\mathbf{T}_{b}\left(\boldsymbol{\Theta}_{n b}\right) \boldsymbol{\omega}_{n b}^{b} .
$$

As in the case of the linear velocities,

$$
\int_{0}^{t} \omega_{n b}^{b} d \tau
$$

has no physical meaning. The orientation of the vessel is obtained by integrating (40).

\subsection{Kinematic Model $\{n\}-\{b\}$}

Following the notation introduced by Fossen (2002) we can define the generalised coordinate position vector (position-orientation vector):

$$
\boldsymbol{\eta} \triangleq\left[\begin{array}{c}
\mathbf{r}_{n b}^{n} \\
\boldsymbol{\Theta}_{n b}
\end{array}\right]=[N, E, D, \phi, \theta, \psi]^{T} .
$$

and the generalised velocity vector (linear-angular velocity vector) in $\{b\}$ is defined as:

$$
\boldsymbol{\nu} \triangleq\left[\begin{array}{c}
\mathbf{v}_{n b}^{b} \\
\boldsymbol{\omega}_{n b}^{b}
\end{array}\right]=[u, v, w, p, q, r]^{T}
$$

From the definition of these two vectors and (37) and (40), the manoeuvring kinematic model follows:

$$
\dot{\boldsymbol{\eta}}=\mathbf{J}_{b}^{n}(\boldsymbol{\eta}) \boldsymbol{\nu}
$$

with

$$
\mathbf{J}_{b}^{n}(\boldsymbol{\eta}) \triangleq\left[\begin{array}{cc}
\mathbf{R}_{b}^{n}\left(\boldsymbol{\Theta}_{n b}\right) & \mathbf{0}_{3 \times 3} \\
\mathbf{0}_{3 \times 3} & \mathbf{T}_{b}\left(\boldsymbol{\Theta}_{n b}\right)
\end{array}\right]
$$

Note that

$$
\mathbf{J}_{b}^{n}(\boldsymbol{\eta})^{-1} \triangleq \mathbf{J}_{n}^{b}(\boldsymbol{\eta})=\left[\begin{array}{cc}
\mathbf{R}_{n}^{b}\left(\boldsymbol{\Theta}_{n b}\right) & \mathbf{0}_{3 \times 3} \\
\mathbf{0}_{3 \times 3} & \mathbf{T}_{b}^{-1}\left(\boldsymbol{\Theta}_{n b}\right)
\end{array}\right],
$$

and $\mathbf{J}_{b}^{n}(\boldsymbol{\eta})^{-1} \neq \mathbf{J}_{b}^{n}(\boldsymbol{\eta})^{T}$ because $\mathbf{T}_{b}$ is not orthogonal.

\section{Seakeeping Kinematics}

\subsection{Coordinate Systems and Motion Variables}

In seakeeping, the motion of the vessel is described relative to the seakeeping coordinate system $\{s\}$, which is an inertial coordinate system fixed to an equilibrium. In the absence of wave excitation forces, the vessel remains in this equilibrium. When there is wave excitation, however, the vessel oscillates with respect to this equilibrium. Figure 2 illustrates this. The equilibrium vessel state is defined by a constant heading and speed:

$$
\begin{aligned}
\mathbf{v}_{n s}^{n} & =\dot{\mathbf{r}}_{n s}^{n}=[U \cos \bar{\psi}, U \sin \bar{\psi}, 0]^{T} \\
\boldsymbol{\omega}_{n s}^{n} & =[0,0,0]^{T}, \\
\boldsymbol{\Theta}_{n s} & =[0,0, \bar{\psi}]^{T},
\end{aligned}
$$

where the velocity $U=\left\|\mathbf{v}_{n s}^{n}\right\|=\left\|^{n} d \vec{r}_{n s} / d t\right\|$ and the heading $\bar{\psi}$ are constant. Hence, the velocity of $\{s\}$ in $\{n\}$ expressed in $\{s\}$ is

$$
\mathbf{v}_{n s}^{s}=\mathbf{R}_{n}^{s} \mathbf{v}_{n s}^{n}=[U, 0,0]^{T}
$$

In a similar fashion as we did for manoeuvring kinematics, we can consider the linear and angular velocity of the vessel $(\{b\})$ relative to $\{s\}$ expressed in $\{b\}$ :

$$
\begin{aligned}
& \mathbf{v}_{s b}^{b}=\mathbf{R}_{s}^{b} \dot{\mathbf{r}}_{s b}^{s} \triangleq[\delta u, \delta v, \delta w]^{T}, \\
& \boldsymbol{\omega}_{s b}^{b} \triangleq[\delta p, \delta q, \delta r]^{T},
\end{aligned}
$$

where these are the perturbation body-fixed linear and angular velocities. The Euler angles that take $\{s\}$ into $\{b\}$ will be denoted by

$$
\boldsymbol{\Theta}_{s b} \triangleq[\delta \phi, \delta \theta, \delta \psi]^{T}
$$

and then from (28) the following relation holds

$$
\dot{\boldsymbol{\Theta}}_{s b}=\mathbf{T}_{b}\left(\boldsymbol{\Theta}_{s b}\right) \boldsymbol{\omega}_{s b}^{b}
$$

with

$$
\mathbf{T}_{b}\left(\boldsymbol{\Theta}_{s b}\right)=\left[\begin{array}{ccc}
1 & s_{\delta \phi} t_{\delta \theta} & c_{\delta \phi} t_{\delta \theta} \\
0 & c_{\delta \phi} & -s \delta \phi \\
0 & s_{\delta \phi} / c_{\delta \theta} & c_{\delta \phi} / c_{\delta \theta}
\end{array}\right]
$$

Further, we can define the perturbation generalised position and velocity vectors:

$$
\delta \boldsymbol{\eta} \triangleq\left[\begin{array}{c}
\mathbf{r}_{s b}^{s} \\
\mathbf{\Theta}_{s b}
\end{array}\right], \quad \delta \boldsymbol{\nu} \triangleq\left[\begin{array}{c}
\mathbf{v}_{s b}^{b} \\
\boldsymbol{\omega}_{s b}^{b}
\end{array}\right] .
$$

In the hydrodynamic literature, the following variables are commonly used:

$$
\xi \triangleq \delta \boldsymbol{\eta}
$$

where $\xi_{1}, \xi_{2}, \xi_{3}$ are the surge, sway and heave perturbation displacements, and $\xi_{4}, \xi_{5}, \xi_{6}$ are the roll, pitch and yaw perturbation angles. 


\subsection{Kinematic Models $\{s\}-\{b\}$}

From the definition of $\delta \boldsymbol{\eta}$ and $\delta \boldsymbol{\nu}$ in (52), it follows that

$$
\delta \dot{\boldsymbol{\eta}}=\mathbf{J}_{b}^{s}(\delta \boldsymbol{\eta}) \delta \boldsymbol{\nu}
$$

where

$$
\mathbf{J}_{b}^{s}(\delta \boldsymbol{\eta})=\left[\begin{array}{cc}
\mathbf{R}_{b}^{s}\left(\boldsymbol{\Theta}_{s b}\right) & \mathbf{0}_{3 \times 3} \\
\mathbf{0}_{3 \times 3} & \mathbf{T}_{b}\left(\boldsymbol{\Theta}_{s b}\right)
\end{array}\right] .
$$

This kinematic model is similar to that for the generalised variables used in manoeuvring, i.e., (43). This is an expected result because both $\{n\}$ and $\{s\}$ are assumed inertial.

The other kinematic models of interest are those that describe the relation between $\boldsymbol{\nu}$ and $\delta \boldsymbol{\nu}$ and between $\dot{\boldsymbol{\nu}}$ and $\delta \dot{\boldsymbol{\nu}}$. To obtain these models, let us consider Figure 2. From this figure, it follows that

$$
\vec{r}_{n b}=\vec{r}_{n s}+\vec{r}_{s b}
$$

By expressing this in coordinate form in $\{n\}$, we obtain

$$
\mathbf{r}_{n b}^{n}=\mathbf{r}_{n s}^{n}+\mathbf{R}_{s}^{n} \mathbf{r}_{s b}^{s} .
$$

Taking the time derivative gives

$$
\dot{\mathbf{r}}_{n b}^{n}=\dot{\mathbf{r}}_{n s}^{n}+\mathbf{R}_{s}^{n} \dot{\mathbf{r}}_{s b}^{s} .
$$

Note that the time-derivative of $\mathbf{R}_{s}^{n}$ is zero because $\{s\}$ does not rotate with respect to $\{n\}$. Further, the last expression can be written as

$$
\begin{aligned}
\dot{\mathbf{r}}_{n b}^{n} & =\dot{\mathbf{r}}_{n s}^{n}+\mathbf{R}_{s}^{n} \mathbf{R}_{b}^{s} \dot{\mathbf{r}}_{s b}^{b}, \\
& =\dot{\mathbf{r}}_{n s}^{n}+\mathbf{R}_{b}^{n} \dot{\mathbf{r}}_{s b}^{b} .
\end{aligned}
$$

If we express the latter in $\{b\}$, we obtain the sought relationship for the linear velocities:

$$
\begin{aligned}
\mathbf{R}_{n}^{b} \dot{\mathbf{r}}_{n b}^{n} & =\mathbf{R}_{n}^{b} \dot{\mathbf{r}}_{n s}^{n}+\mathbf{R}_{n}^{b} \mathbf{R}_{b}^{n} \dot{\mathbf{r}}_{s b}^{b}, \\
\mathbf{v}_{n b}^{b} & =\mathbf{R}_{n}^{b} \mathbf{v}_{n s}^{n}+\mathbf{v}_{s b}^{b} .
\end{aligned}
$$

Let us split the linear and angular part of the velocity vectors:

$$
\boldsymbol{\nu}=\left[\begin{array}{l}
\boldsymbol{\nu}_{1} \\
\boldsymbol{\nu}_{2}
\end{array}\right], \quad \delta \boldsymbol{\nu}=\left[\begin{array}{l}
\delta \boldsymbol{\nu}_{1} \\
\delta \boldsymbol{\nu}_{2}
\end{array}\right],
$$

where $\boldsymbol{\nu}_{1}=[u, v, w]^{T}, \quad \boldsymbol{\nu}_{2}=[p, q, r]^{T}, \quad \delta \boldsymbol{\nu}_{1}=$ $[\delta u, \delta v, \delta w]^{T}$, and $\delta \boldsymbol{\nu}_{2}=[\delta p, \delta q, \delta r]^{T}$. Then from (60) it follows that

$$
\boldsymbol{\nu}_{1}=\overline{\boldsymbol{\nu}}_{1}+\delta \boldsymbol{\nu}_{1}
$$

where

$$
\overline{\boldsymbol{\nu}}_{1} \triangleq \mathbf{R}_{n}^{b}\left[\begin{array}{c}
U \cos \bar{\psi} \\
U \sin \bar{\psi} \\
0
\end{array}\right]=\mathbf{R}_{s}^{b}\left[\begin{array}{l}
U \\
0 \\
0
\end{array}\right] .
$$

To obtain the angular velocity transformation, we can turn again to Figure 2 and from (19), it follows that

$$
\vec{\omega}_{n b}=\vec{\omega}_{n s}+\vec{\omega}_{s b}
$$

But since $\{s\}$ does not rotate with respect to $\{n\}$,

$$
\vec{\omega}_{n b}=\vec{\omega}_{s b}
$$

This leads to the sought relationship for the angular velocities

$$
\boldsymbol{\omega}_{n b}^{b}=\boldsymbol{\omega}_{s b}^{b} \Rightarrow \boldsymbol{\nu}_{2}=\delta \boldsymbol{\nu}_{2} .
$$

Combining (62) and (66), we finally obtain

$$
\boldsymbol{\nu}=\overline{\boldsymbol{\nu}}+\delta \boldsymbol{\nu},
$$

where $\overline{\boldsymbol{\nu}}=\left[\overline{\boldsymbol{\nu}}_{1}^{T}, \mathbf{0}_{3 \times 1}\right]^{T}$ and $\overline{\boldsymbol{\nu}}_{1}$ is given in (63).

Further, from (63) it follows that

$$
\begin{aligned}
\overline{\boldsymbol{\nu}}_{1} & =U \operatorname{col}_{1}\left(\mathbf{R}_{s}^{b}\right) \\
& =U\left[\begin{array}{c}
c_{\delta \psi} c_{\delta \theta} \\
-s_{\delta \psi} c_{\delta \theta}+c_{\delta \psi} s_{\delta \theta} s_{\delta \phi} \\
s_{\delta \psi} s_{\delta \phi}+c_{\delta \psi} c_{\delta \phi} s_{\delta \theta}
\end{array}\right],
\end{aligned}
$$

where $\operatorname{col}_{j}(\mathbf{R})$ denotes the $j$-column of the matrix $\mathbf{R}$. Taking small angle approximations and considering only linear terms, we obtain

$$
\overline{\boldsymbol{\nu}}_{1} \approx U\left[\begin{array}{c}
1 \\
-\delta \psi \\
\delta \theta
\end{array}\right]
$$

Hence, (67) can be approximated by

$$
\boldsymbol{\nu} \approx U\left(-\mathbf{L} \delta \boldsymbol{\eta}+\mathbf{e}_{1}\right)+\delta \boldsymbol{\nu}
$$

with

$$
\mathbf{e}_{1} \triangleq[1,0, \ldots, 0]^{T}, \quad \mathbf{L} \triangleq\left[\begin{array}{cccc}
0 & \cdots & 0 & 0 \\
0 & \cdots & 0 & 1 \\
0 & \cdots & -1 & 0 \\
\vdots & \ddots & \vdots & \vdots \\
0 & \cdots & 0 & 0
\end{array}\right] .
$$

To obtain the relation between accelerations we take the time derivative of $(67)$ :

$$
\dot{\nu}=\dot{\bar{\nu}}+\delta \dot{\nu} .
$$

The key issue here is to find $\dot{\bar{\nu}}$. From (63) it follows that

$$
\begin{aligned}
\dot{\bar{\nu}}_{1} & =\mathbf{R}_{s}^{b} \mathbf{S}^{T}\left(\boldsymbol{\omega}_{s b}^{b}\right)\left[\begin{array}{c}
U \\
0 \\
0
\end{array}\right]=\mathbf{R}_{s}^{b} U\left[\begin{array}{c}
0 \\
-\delta r \\
\delta q
\end{array}\right] \\
& =-U \delta r \operatorname{col}_{2}\left(\mathbf{R}_{s}^{b}\right)+U \delta q \operatorname{col}_{3}\left(\mathbf{R}_{s}^{b}\right) .
\end{aligned}
$$

Taking small angle approximations

$$
\dot{\tilde{\boldsymbol{\nu}}}_{1} \approx-U \delta r\left[\begin{array}{c}
\delta \psi \\
1 \\
-\delta \phi
\end{array}\right]+U \delta q\left[\begin{array}{c}
-\delta \theta \\
\delta \phi \\
1
\end{array}\right]
$$


If we consider only the linear terms

$$
\dot{\bar{\nu}}_{1} \approx U\left[\begin{array}{c}
0 \\
-\delta r \\
\delta q
\end{array}\right]=-U \mathbf{L} \delta \boldsymbol{\nu}
$$

which is consistent with the approximation (69) taking $\delta \dot{\boldsymbol{\eta}} \approx \delta \boldsymbol{\nu}$. Hence, using (75), it follows that (72) can be approximated as

$$
\dot{\nu} \approx-U \mathbf{L} \delta \boldsymbol{\nu}+\dot{\delta} \boldsymbol{\nu}
$$

Finally, we can also relate $\delta \boldsymbol{\nu}$ to the vessel trajectory. Indeed, from (59) it follow that

$$
\dot{\boldsymbol{\eta}}_{1}=\left[\begin{array}{c}
U \cos \bar{\psi} \\
U \sin \bar{\psi} \\
0
\end{array}\right]+\mathbf{R}_{b}^{n} \delta \boldsymbol{\nu}_{1}
$$

Also, from (40) and (65) it follows that

$$
\begin{aligned}
\dot{\boldsymbol{\eta}}_{2}=\dot{\boldsymbol{\Theta}}_{n b} & =\mathbf{T}_{d}\left(\boldsymbol{\Theta}_{n b}\right) \boldsymbol{\omega}_{n b}^{b} \\
& =\mathbf{T}_{d}\left(\boldsymbol{\Theta}_{n b}\right) \boldsymbol{\omega}_{s b}^{b} \\
& =\mathbf{T}_{d}\left(\boldsymbol{\Theta}_{n b}\right) \delta \boldsymbol{\nu}_{2}
\end{aligned}
$$

Therefore,

$$
\dot{\boldsymbol{\eta}}=\left[\begin{array}{c}
U \cos \bar{\psi} \\
U \sin \bar{\psi} \\
\mathbf{0}_{4 \times 1}
\end{array}\right]+\mathbf{J}_{b}^{n}(\boldsymbol{\eta}) \delta \boldsymbol{\nu}
$$

\subsection{Relationship between Euler Angles}

The relationship between the Euler angles that take $\{n\}$ into $\{b\}$ and those that take $\{s\}$ into $\{b\}$ is as follows. From the seakeeping assumption we have that

$$
\overrightarrow{\boldsymbol{\omega}}_{n b}=\overrightarrow{\boldsymbol{\omega}}_{s b} \quad \Rightarrow \quad \boldsymbol{\omega}_{n b}^{b}=\boldsymbol{\omega}_{s b}^{b}
$$

which results in

$$
\dot{\boldsymbol{\Theta}}_{n b}=\dot{\boldsymbol{\Theta}}_{s b} \text {. }
$$

Integrating, we obtain

$$
\boldsymbol{\Theta}_{n b}=\boldsymbol{\Theta}_{s b}+\boldsymbol{\Theta}_{n s}
$$

that is,

$$
\left[\begin{array}{l}
\phi \\
\theta \\
\psi
\end{array}\right]=\left[\begin{array}{l}
\delta \phi \\
\delta \theta \\
\delta \psi
\end{array}\right]+\left[\begin{array}{l}
0 \\
0 \\
\bar{\psi}
\end{array}\right]
$$

Tables 1, 2 and 3 in the Appendix summarize the notation.

\section{Application to Equations of Motion Transformation}

In recent years, there has been a significant interest in time-domain models for simulation and control system design based on data obtained from seakeeping programs - see Bailey et al. (1997); Fossen and Smogeli (2004); Perez (2005); Fossen (2005); Smogeli et al. (2005). This requires transforming the equations of motion used in seakeeping theory, (or at least the forces) to body-fixed coordinates. These transformations can be done in different ways using different approximations. This is the topic of this section, which makes use of the kinematic models developed in the previous sections of the paper

\subsection{Rigid-body Mass and Inertia Tensor}

The vessel rigid-body generalized mass matrix about the origin of $\{b\}$ is of the form

$$
\mathbf{M}_{R B}^{b}=\left[\begin{array}{cc}
m \mathbf{I}_{3 \times 3} & -m \mathbf{S}\left(\mathbf{r}_{b g}^{b}\right) \\
m \mathbf{S}\left(\mathbf{r}_{b g}^{b}\right) & \mathbf{I}_{b / b}^{b}
\end{array}\right]
$$

where $m$ is the mass of the vessel and $\mathbf{I}_{b / b}^{b}$ is the inertia tensor about $(b /) o_{b}$. Using the parallel-axis theorem (see for example Egeland and Gravdahl (2002)), this tensor can be expressed as

$$
\mathbf{I}_{b / b}^{b}=\mathbf{I}_{b / g}^{b}-m \mathbf{S}\left(\mathbf{r}_{b g}^{b}\right) \mathbf{S}\left(\mathbf{r}_{b g}^{b}\right),
$$

where $\mathbf{r}_{b g}^{b}$ gives the coordinates of the centre of gravity $(\mathrm{CG})$ in $\{b\}$, and $\mathbf{I}_{b / g}^{b}$ is the tensor about GG in $\{b\}$ :

$$
\mathbf{I}_{b / g}^{b}=\int_{b}\left[\begin{array}{ccc}
y^{2}+z^{2} & -x y & -x z \\
-x y & x^{2}+z^{2} & -y z \\
-x z & -y z & x^{2}+y^{2}
\end{array}\right] d m .
$$

The angular momentum of the vessel about CG in $\{b\}$ is given by

$$
\mathbf{h}_{g}^{b}=\mathbf{I}_{b / g}^{b} \boldsymbol{\omega}_{i b}^{b},
$$

where $\{i\}$ represents any inertial frame (e.g., $\{n\}$ or $\{s\})$. If this is transformed to an inertial coordinate system, then

$$
\mathbf{h}_{g}^{i}=\mathbf{R}_{b}^{i} \mathbf{I}_{b / g}^{b} \mathbf{R}_{i}^{b} \boldsymbol{\omega}_{i b}^{i}
$$

from which it follows that

$$
\mathbf{I}_{b / g}^{i}=\mathbf{R}_{b}^{i} \mathbf{I}_{b / g}^{b} \mathbf{R}_{i}^{b} .
$$

This shows that the inertia tensor will not be constant in an inertial frame if $\{b\}$ rotates with respect to $\{i\}$. Therefore, it is always convenient to formulate the equations of motion in a body-fixed rather than inertial coordinate system. 


\subsection{Rigid-body Equations of Motion}

Following Fossen (2002), the rigid-body equations of motion in $\{b\}$ can be written as

$$
\mathbf{M}_{R B}^{b} \dot{\boldsymbol{\nu}}+\mathbf{C}_{R B}(\boldsymbol{\nu}) \boldsymbol{\nu}=\tau^{b}
$$

where $\mathbf{M}_{R B}^{b}$ is given in (84), $\boldsymbol{\nu}$ is given in (42), and the Coriolis-Centripetal matrix $\mathbf{C}_{R B}(\boldsymbol{\nu})$ can be expressed as:

$$
\mathbf{C}_{R B}(\boldsymbol{\nu}) \triangleq\left[\begin{array}{ll}
\mathbf{C}_{R B, 11} & \mathbf{C}_{R B, 12} \\
\mathbf{C}_{R B, 21} & \mathbf{C}_{R B, 22}
\end{array}\right]
$$

where

$$
\begin{aligned}
& \mathbf{C}_{R B, 11}=\mathbf{0}_{3 \times 3}, \\
& \mathbf{C}_{R B, 12}=-m \mathbf{S}\left(\boldsymbol{\nu}_{1}\right)-m \mathbf{S}\left(\mathbf{S}\left(\boldsymbol{\nu}_{2}\right) \mathbf{r}_{b g}^{b}\right), \\
& \mathbf{C}_{R B, 21}=-m \mathbf{S}\left(\boldsymbol{\nu}_{1}\right)-m \mathbf{S}\left(\mathbf{S}\left(\boldsymbol{\nu}_{2}\right) \mathbf{r}_{b g}^{b}\right), \\
& \mathbf{C}_{R B, 22}=m \mathbf{S}\left(\mathbf{S}\left(\boldsymbol{\nu}_{1}\right) \mathbf{r}_{b g}^{b}\right)-\mathbf{S}\left(\mathbf{I}_{b / b}^{b} \boldsymbol{\nu}_{2}\right),
\end{aligned}
$$

with $\boldsymbol{\nu}=\left[\boldsymbol{\nu}_{1}^{T}, \boldsymbol{\nu}_{2}^{T}\right]^{T}$. See Fossen (2002) for further details and alternative parameterizations of $\mathbf{C}_{R B}(\boldsymbol{\nu})$.

\subsection{Equation of Motion in $\{p\}$}

The equation of motion (90) is formulated at the origin $o_{b}$ of $\{b\}$. In order to exploit different locations of the body-fixed coordinate system, we can transform (90) to another body-fixed coordinate system.

Let $\{p\}$ be a body-fixed coordinate system with the same orientation as $\{b\}$. Then,

$$
\vec{r}_{n p}=\vec{r}_{n b}+\vec{r}_{b p}
$$

The time-derivative in $\{n\}$ give

$$
\dot{\mathbf{r}}_{n p}^{n}=\dot{\mathbf{r}}_{n b}^{n}+\dot{\mathbf{R}}_{b}^{n} \mathbf{r}_{b p}^{b},
$$

which can be written as

$$
\mathbf{v}_{n p}^{n}=\mathbf{v}_{n b}^{n}+\mathbf{R}_{b}^{n} \mathbf{S}\left(\boldsymbol{\omega}_{n b}^{b}\right) \mathbf{r}_{b p}^{b} .
$$

Using the fact that $\{p\}$ has the same orientation as $\{b\}$, i.e., $\mathbf{R}_{b}^{n}=\mathbf{R}_{p}^{n}$, we can further write

$$
\begin{aligned}
\mathbf{v}_{n p}^{p} & =\mathbf{v}_{n b}^{b}+\mathbf{S}\left(\boldsymbol{\omega}_{n b}^{b}\right) \mathbf{r}_{b p}^{b} \\
& =\mathbf{v}_{n b}^{b}+\mathbf{S}^{T}\left(\mathbf{r}_{b p}^{b}\right) \boldsymbol{\omega}_{n b}^{b} .
\end{aligned}
$$

Hence,

$$
\left[\begin{array}{c}
\mathbf{v}_{n p}^{p} \\
\boldsymbol{\omega}_{n p}^{p}
\end{array}\right]=\left[\begin{array}{cc}
\mathbf{0} & \mathbf{S}^{T}\left(\mathbf{r}_{b p}^{b}\right) \\
\mathbf{0} & \mathbf{I}
\end{array}\right]\left[\begin{array}{c}
\mathbf{v}_{n b}^{b} \\
\boldsymbol{\omega}_{n b}^{b}
\end{array}\right],
$$

or equivalently

$$
\boldsymbol{\nu}^{p}=\mathbf{H}\left(\mathbf{r}_{b p}^{b}\right) \boldsymbol{\nu}
$$

with

$$
\begin{aligned}
\mathbf{H}\left(\mathbf{r}_{b p}^{b}\right) & \triangleq\left[\begin{array}{cc}
\mathbf{0}_{3 \times 3} & \mathbf{S}^{T}\left(\mathbf{r}_{b p}^{b}\right) \\
\mathbf{0}_{3 \times 3} & \mathbf{I}_{3 \times 3}
\end{array}\right] \\
\mathbf{H}^{-1}\left(\mathbf{r}_{b p}^{b}\right) & \triangleq\left[\begin{array}{cc}
\mathbf{0}_{3 \times 3} & \mathbf{S}\left(\mathbf{r}_{b p}^{b}\right) \\
\mathbf{0}_{3 \times 3} & \mathbf{I}_{3 \times 3}
\end{array}\right] .
\end{aligned}
$$

If $\vec{f}$ is a force, then the moments about $o_{b}$ and $o_{p}$ are related as follows:

$$
\vec{m}_{p}=\vec{m}_{b}+\vec{r}_{p b} \times \vec{f}=\vec{m}_{b}-\vec{r}_{b p} \times \vec{f} .
$$

Then, in coordinate form

$$
\left[\begin{array}{c}
\mathbf{f}^{p} \\
\mathbf{m}_{p}^{p}
\end{array}\right]=\left[\begin{array}{cc}
\mathbf{I}_{3 \times 3} & \mathbf{0}_{3 \times 3} \\
\mathbf{S}^{T}\left(\mathbf{r}_{b p}^{b}\right) & \mathbf{0}_{3 \times 3}
\end{array}\right]\left[\begin{array}{c}
\mathbf{f}^{b} \\
\mathbf{m}_{b}^{b}
\end{array}\right] .
$$

Therefore,

$$
\boldsymbol{\tau}^{p}=\mathbf{H}^{-T}\left(\mathbf{r}_{b p}^{b}\right) \boldsymbol{\tau} .
$$

Using (102) and (98), the transformation of (90) to $\{p\}$ becomes

$$
\mathbf{M}_{R B}^{p} \dot{\boldsymbol{\nu}}^{p}+\mathbf{C}_{R B}^{p}\left(\boldsymbol{\nu}^{p}\right) \boldsymbol{\nu}^{p}=\boldsymbol{\tau}^{p}
$$

with

$$
\begin{aligned}
\boldsymbol{\nu}^{p} & =\mathbf{H}^{-1}\left(\mathbf{r}_{b p}^{b}\right) \boldsymbol{\nu} \\
\boldsymbol{\tau}^{p} & =\mathbf{H}^{-T}\left(\mathbf{r}_{b p}^{b}\right) \boldsymbol{\tau} \\
\mathbf{M}_{R B}^{p} & =\mathbf{H}^{-T}\left(\mathbf{r}_{b p}^{b}\right) \mathbf{M}_{R B}^{b} \mathbf{H}^{-1}\left(\mathbf{r}_{b p}^{b}\right) \\
\mathbf{C}_{R B}^{p} & =\mathbf{H}^{-T}\left(\mathbf{r}_{b p}^{b}\right) \mathbf{C}_{R B} \mathbf{H}^{-1}\left(\mathbf{r}_{b p}^{b}\right)
\end{aligned}
$$

\subsection{Seakeeping Equations of Motion in $\{s\}$}

Using the perturbation body-fixed coordinates $\delta \boldsymbol{\nu}$ and $\delta \boldsymbol{\eta}$, the seakeeping equations of motion can be formulated as

$$
\begin{aligned}
& \delta \dot{\boldsymbol{\eta}}=\mathbf{J}_{b}^{s}(\delta \boldsymbol{\eta}) \delta \boldsymbol{\nu} \\
& \mathbf{M}_{R B} \delta \dot{\boldsymbol{\nu}}+\mathbf{C}_{R B}(\delta \boldsymbol{\nu}) \delta \boldsymbol{\nu}=\delta \boldsymbol{\tau}
\end{aligned}
$$

where

$$
\mathbf{M}_{R B} \triangleq \mathbf{M}_{R B}^{b} .
$$

The generalised force vector is separated into two components:

$$
\delta \boldsymbol{\tau}=\delta \boldsymbol{\tau}_{\mathrm{rad}}^{b}+\delta \boldsymbol{\tau}_{\mathrm{exc}}^{b},
$$

where $\delta \boldsymbol{\tau}_{\text {rad }}^{s}$ is the vector of radiation forces, and $\delta \boldsymbol{\tau}_{\text {exc }}^{s}$ is the vector of excitation forces which include the environmental and control forces.

If we consider the motion within a linear framework, then (105) becomes

$$
\begin{aligned}
\delta \dot{\boldsymbol{\eta}} & \approx \delta \boldsymbol{\nu} \\
\mathbf{M}_{R B} \delta \dot{\boldsymbol{\nu}} & \approx \delta \boldsymbol{\tau}
\end{aligned}
$$

which results in

$$
\mathbf{M}_{R B} \delta \ddot{\boldsymbol{\eta}} \approx \delta \boldsymbol{\tau}
$$

In seakeeping theory this equation is normally written as

$$
\mathbf{M}_{R B} \ddot{\boldsymbol{\xi}}=\boldsymbol{\tau}_{\mathrm{rad}}^{s}+\boldsymbol{\tau}_{\mathrm{exc}}^{s}
$$


where $\boldsymbol{\xi}=\delta \boldsymbol{\eta}$. Equation (109) is a convenient and consistent way to write (108) with all the variables in $\{s\}$-however, it should be noted that it arises from the following approximations:

$$
\begin{aligned}
\dot{\boldsymbol{\xi}}=\delta \dot{\boldsymbol{\eta}} & \approx \delta \boldsymbol{\nu}, \\
\boldsymbol{\tau}_{\mathrm{rad}}^{s} & \approx \delta \boldsymbol{\tau}_{\mathrm{rad}}^{b}, \\
\boldsymbol{\tau}_{\mathrm{exc}}^{s} & \approx \delta \boldsymbol{\tau}_{\mathrm{exc}}^{b} .
\end{aligned}
$$

Following Cummins (1962), the radiation forces in $\{s\}$ can be expressed as

$$
\begin{aligned}
\boldsymbol{\tau}_{\text {rad }}^{s}=-\overline{\mathbf{A}} \ddot{\boldsymbol{\xi}}-\overline{\mathbf{B}} \dot{\boldsymbol{\xi}} & \\
& -\int_{0}^{t} \mathbf{K}\left(t-t^{\prime}\right) \dot{\boldsymbol{\xi}}\left(t^{\prime}\right) d t^{\prime}-\mathbf{G} \boldsymbol{\xi},
\end{aligned}
$$

where $\overline{\mathbf{A}}=\lim _{\omega \rightarrow \infty} \mathbf{A}(\omega)$ and $\overline{\mathbf{B}}=\lim _{\omega \rightarrow \infty} \mathbf{B}(\omega)$ are the constant infinite frequency added mass and potential damping matrices, $\mathbf{G}$ is the restoring matrix, and $\mathbf{K}(t)$ are retardation functions (Ogilvie, 1964):

$$
\mathbf{K}(t)=\int_{0}^{\infty}(\mathbf{B}(\omega)-\overline{\mathbf{B}}) \cos (\omega t) d \omega .
$$

The matrix $\overline{\mathbf{B}}$ vanishes for the case of zero forward speed. Substituting (111) into (109) leads to the linear seakeeping vector equation of motion (Cummins Equation):

$$
\begin{aligned}
\left(\mathbf{M}_{R B}+\right. & \overline{\mathbf{A}}) \ddot{\boldsymbol{\xi}}+\overline{\mathbf{B}} \dot{\boldsymbol{\xi}} \\
& +\int_{0}^{t} \mathbf{K}\left(t-t^{\prime}\right) \dot{\boldsymbol{\xi}}\left(t^{\prime}\right) d t^{\prime}+\mathbf{G} \boldsymbol{\xi}=\boldsymbol{\tau}_{\text {exc }}^{s}
\end{aligned}
$$

The convolution term represents the fluid memory effect; that is, the change in fluid momentum due to the motion of the hull at a particular time instant affects the motion of the vessel at all subsequent times.

\subsection{Seakeeping Equations of Motion in $\{b\}$}

In order to design control systems, it is convenient to express (112) in $\{b\}$ rather than in $\{s\}$. Hydrodynamic programs compute $\boldsymbol{\tau}_{\text {rad }}^{s}$ and $\boldsymbol{\tau}_{\text {exc }}^{s}$ based on potential theory. These forces are calculated by integrating the pressure of the fluid over the wetted surface of the hull. The reason for using the $\{s\}$ coordinate system is because these forces are computed for the vessel in its equilibrium position. To express these forces in $\{b\}$, we would have to rotate them. However, this would result in a time varying added mass matrix. Therefore, from (110), it is common to approximate the radiation forces in $\{b\}$ as

$$
\begin{aligned}
& \delta \boldsymbol{\tau}_{r a d}^{b} \approx-\overline{\mathbf{A}} \delta \dot{\boldsymbol{\nu}}-\overline{\mathbf{B}} \delta \boldsymbol{\nu} \\
&-\int_{0}^{t} \mathbf{K}\left(t-t^{\prime}\right) \delta \boldsymbol{\nu}\left(t^{\prime}\right) d t^{\prime}-\mathbf{G} \delta \boldsymbol{\eta} .
\end{aligned}
$$

Substituting (113) into (107) we obtain the linear perturbation model in $\{b\}$ :

$$
\mathbf{M} \delta \dot{\boldsymbol{\nu}}+\overline{\mathbf{B}} \delta \boldsymbol{\nu}+\int_{0}^{t} \mathbf{K}\left(t-t^{\prime}\right) \delta \boldsymbol{\nu}\left(t^{\prime}\right) d t^{\prime}+\mathbf{G} \delta \boldsymbol{\eta}=\delta \boldsymbol{\tau}_{\mathrm{exc}}^{b}
$$

where $\mathbf{M} \triangleq \mathbf{M}_{R B}+\overline{\mathbf{A}}$. This model describes deviations from the equilibrium state. Using the small angle transformations (70) and (76), we can express (114) as

$$
\begin{aligned}
\dot{\boldsymbol{\eta}} & =\mathbf{J}_{b}^{n}(\boldsymbol{\eta}) \boldsymbol{\nu} \\
\mathbf{M} \dot{\boldsymbol{\nu}} & +\mathbf{C}_{R B} \boldsymbol{\nu}+\mathbf{C}_{A} \boldsymbol{\nu}+\overline{\mathbf{B}} \boldsymbol{\nu} \\
& +\int_{0}^{t} \mathbf{K}\left(t-t^{\prime}\right)\left[\boldsymbol{\nu}\left(t^{\prime}\right)+U \mathbf{L} \boldsymbol{\eta}\left(t^{\prime}\right)\right] d t^{\prime} \\
& +\mathbf{G} \boldsymbol{\eta}=\boldsymbol{\tau}_{\mathrm{exc}}^{b}+\overline{\boldsymbol{\tau}}^{b}
\end{aligned}
$$

where

$$
\begin{aligned}
\mathbf{C}_{R B} & \triangleq \mathbf{M}_{R B} U \mathbf{L}, \\
\mathbf{C}_{A} & \triangleq \overline{\mathbf{A}} U \mathbf{L}, \\
\overline{\boldsymbol{\tau}}^{b} & \triangleq \overline{\mathbf{B}} \overline{\boldsymbol{\nu}} .
\end{aligned}
$$

Expressions (115) give the linear equations of motion with fluid memory effects for constant average forward speed and heading. These equations are valid for small deviations from the equilibrium heading. Note that most strip-theory codes usually incorporate $\mathbf{C}_{A}$ in $\overline{\mathbf{B}}$; therefore in these cases, $\mathbf{C}_{A}$ should be set to zero so as not incorporate the terms twice in (115). If a motion control system is designed, the control forces will have two components, e.g., $\boldsymbol{\tau}_{c}^{b}=\overline{\boldsymbol{\tau}}^{b}+\boldsymbol{\tau}_{P I D}^{b}$, where the first term is the force that sets the equilibrium, and the second one corrects the deviations from it.

For the case of station keeping or manoeuvring at low speed, these reduce to

$$
\begin{aligned}
\mathbf{M} \dot{\boldsymbol{\nu}}+\int_{0}^{t} \mathbf{K}\left(t-t^{\prime}\right) \boldsymbol{\nu}\left(t^{\prime}\right) d t^{\prime} \boldsymbol{\nu}+\mathbf{G} \boldsymbol{\eta} & =\boldsymbol{\tau}_{\mathrm{exc}}^{b} \\
\dot{\boldsymbol{\eta}} & =\mathbf{J}_{b}^{n}(\boldsymbol{\eta}) \boldsymbol{\nu}
\end{aligned}
$$

Note that (115) and (117) are based on potential theory. Therefore, a viscous damping term $\mathbf{B}_{v} \boldsymbol{\nu}$ can be added to the left-hand side of (115) and (117) - see Bailey et al. (1997); Fossen and Smogeli (2004); Fossen (2005); Smogeli et al. (2005); Perez (2005); Perez and Fossen (2006).

\section{Conclusions}

In this paper, the kinematic models most commonly used for the description of ship motion were derived using the formalism of the robotics literature. The classical transformation used in ship manoeuvring, which relates the body-fixed linear and angular velocity to 
the position and orientation in local geographical coordinates was revisited. The kinematic models used in seakeeping, together with the simplifying assumptions that lead to them, were considered with a notation consistent with that used in manoeuvring. Transformation between variables were derived, and the results were applied to expressing the seakeeping equations of motion in terms of manoeuvring variables. The latter provides the basis for models that can be used in guidance and motion control systems.

\section{Acknowledgments}

The Authors would like to thank Prof. Olav Egeland for his constructive discussions about the presented material. This work was supported by Centre for Ships and Ocean Structures (CeSOS) and its sponsor the Norwegian Research Council.

\section{References}

Bailey, P., Price, W., and Temarel, P. A unified mathematical model describing the manoeuvring of a ship in seaway. Transactions The Royal Institution of Naval Architects-RINA, 1997. 140:131-149.

Bishop, R. and Price, W. On the use of equilibrium axes and body axes in the dynamics of a rigid ship. Journal of Mechanical Engieering Scinece, 1981. 23(5):243-256.

Cummins, W. The impulse response function and ship motion. Technical Report 1661, David Taylor Model Basin-DTNSRDC, 1962.

Egeland, O. and Gravdahl, J. Modeling and Simulation for Automatic Control. Marine Cybernetics, Trondheim, 2002.

Faltinsen, O. Hydrodynamic of High-speed Marine Vehicles. Cambridge University Press, 2005.

Fathi, D. ShipX Vessel Responses (VERES). Marintek AS Trondheim, http://www.marintek.sintef.no/, 2004.

Fossen, T. Marine Control Systems: Guidance, Navigation and Control of Ships, Rigs and Underwater Vehicles. Marine Cybernetics, Trondheim, 2002.

Fossen, T. A nonlinear unified state-space model for ship maneuvreing and control in a seaway. In International Journal of Bifurcation and Chaos, volume 15. 2005 pages 2717-2746.
Fossen, T. and Smogeli, Ø. Nonlinear time-domain strip theory formulation for low speed manoeuvring and station-keeping. Modelling Identification and Control-MIC, 2004. 25(4).

Graham, R. Motion-induced interruptions as ship operability criteria. Naval Engineers Journal, 1990. $103(3)$.

Journee, J. and Adegeest, L. Theoretical Manual of Strip Theory Program SEAWAY for Windows. TU Delft, Delft University of Technology, www.ocp.tudelft.nl/mt/journee, 2003.

Kane, T. and Levinson, D. Dynamics: Theory and Applications. McGraw-Hill series in Mechanical Engineering. McGraw-Hill, 1985.

Lloyd, A. Seakeeping: Ship Behaviour in Rough Weather. Ellis Horwood Series in Marine Technology. Ellis Horwood, 1989.

Ogilvie, T. Recent progress towards the understanding and prediction of ship motions. In 6th Symposium on Naval Hydrodynamics. 1964 .

Perez, T. Ship Motion Control: Course Keeping and Roll Reduction using rudder and fins. Advances in Industrial Control. Springer-Verlag, London, 2005.

Perez, T. and Fossen, T. Time-domain models of marine surface vessels for simulation and control design based on seakeeping computations. In Proc. 7th IFAC Conference on Manoeuvring and Control of Marine Craft MCMC. Lisbon, Portugal, 2006 .

Rao, A. Dynamics of Particles and Rigid Bodies A Systematic Approach. Cambridge University Press, 2006.

Sciavicco, L. and Siciliano, B. Modelling and Control of Robot Manipulators. Advanced Textbooks in control and Signal Processing. Springer-Verlag London (5th printing), 2004.

Smogeli, Ø., Perez, T., Fossen, T., and Sørensen, A. The marine systems simulator state-space model representation for dynamically positioned surface vessels. In International Maritime Association of the Mediterranean IMAM Conference, Lisbon, Portugal. 2005 .

SNAME. Nomenclature for treating the motion of a submerged body through a fluid. Technical Report Bulletin 1-5, Society of Naval Architects and Marine Engineers, New York, USA, 1950.

WAMIT. WAMIT User Manual. www.wamit.com, 2004. 


\section{A Summary of Variables}

Table 1: Scalar variables used the manoeuvring and seakeeping.

\begin{tabular}{lll}
\hline Variable & Name & Frame \\
\hline \hline$N$ & North position & $\{n\}$ \\
$E$ & East position & $\{n\}$ \\
$D$ & Down position & $\{n\}$ \\
\hline$\phi$ & Roll angle & - \\
$\theta$ & Pitch angle & - \\
$\psi$ & Heading or yaw angle & $\{b\}$ \\
\hline$u, \delta u$ & Surge speed, Surge pert. speed & $\{b\}$ \\
$v, \delta v$ & Sway speed, Sway pert. speed & $\{b\}$ \\
$w, \delta w$ & Heave speed, Heave pert. speed & $\{b\}$ \\
\hline$p, \delta p$ & Roll rate, Roll perturbation rate & $\{b\}$ \\
$q, \delta q$ & Pitch rate, Pitch perturbation rate & $\{b\}$ \\
$r, \delta r$ & Yaw rate, Yaw perturbation rate & $\{s\}$ \\
\hline$\xi_{1}$ & Surge perturbation displacement & $\{s\}$ \\
$\xi_{2}$ & Sway perturbation displacement & $\{s\}$ \\
$\xi_{3}$ & Heave perturbation displacement & $\{s\}$ \\
\hline$\xi_{4}=\delta \phi$ & Roll perturbation angle & - \\
$\xi_{5}=\delta \theta$ & Pitch perturbation angle & - \\
$\xi_{6}=\delta \psi$ & Yaw perturbation angle & - \\
\hline
\end{tabular}

Table 2: Summary manoeuvring vector variables.

\begin{tabular}{ll}
\hline Variable & Description \\
\hline \hline $\mathbf{r}_{n b}^{n}=[N, E, D]^{T}$ & Vessel position in $\{n\}$ \\
$\mathbf{v}_{n b}^{b}=[u, v, w]^{T}$ & Vessel linear velocity in $\{b\}$ \\
$\boldsymbol{\omega}_{n b}^{b}=[p, q, r]^{T}$ & Vessel angular velocity in $\{b\}$ \\
$\boldsymbol{\Theta}_{n b}=[\phi, \theta, \psi]^{T}$ & Euler angles that take $\{n\}$ into $\{b\}$ \\
$\boldsymbol{\eta}=\left[\left(\mathbf{r}_{n b}^{n}\right)^{T},\left(\boldsymbol{\Theta}_{n b}\right)^{T}\right]^{T}$ & Generalised position vector \\
$\boldsymbol{\nu}=\left[\left(\mathbf{v}_{n b}^{b}\right)^{T},\left(\boldsymbol{\omega}_{n b}^{b}\right)^{T}\right]^{T}$ & Generalised velocity vector \\
$\dot{\boldsymbol{\eta}}=\mathbf{J}_{b}^{n}(\boldsymbol{\eta}) \boldsymbol{\nu}$ & Vessel trajectory \\
\hline
\end{tabular}

Table 3: Summary seakeeping vector variables.

\begin{tabular}{ll}
\hline Variable & Description \\
\hline \hline $\mathbf{r}_{s b}^{s}$ & Vessel perturbation displ. in $\{s\}$ \\
$\mathbf{v}_{s b}^{b}=[\delta u, \delta v, \delta w]^{T}$ & Vessel linear pert. velocity in $\{b\}$ \\
$\boldsymbol{\omega}_{s b}^{b}=[\delta p, \delta q, \delta r]^{T}$ & Vessel pert. angular vel. in $\{b\}$ \\
$\boldsymbol{\Theta}_{s b}^{b}=[\delta \phi, \delta \theta, \delta \psi]^{T}$ & Euler ang. that take $\{s\}$ into $\{b\}$ \\
$\delta \boldsymbol{\eta}=\left[\left(\mathbf{r}_{s b}^{s}\right)^{T},\left(\boldsymbol{\Theta}_{s b}\right)^{T}\right]^{T}$ & Generalised pert. position vector \\
$\boldsymbol{\xi}=\delta \boldsymbol{\eta}$ & Seakeeping variables \\
$\delta \boldsymbol{\nu}=\left[\left(\mathbf{v}_{s b}^{b}\right)^{T},\left(\boldsymbol{\omega}_{s b}^{b}\right)^{T}\right]^{T}$ & Generalised pert. velocity vector \\
\hline
\end{tabular}

